\title{
Clinical classification of tetanus patients
}

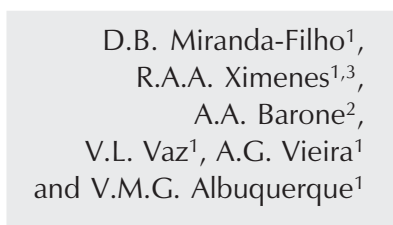

\author{
1Departamento de Medicina Clínica, Faculdade de Ciências Médicas, \\ Universidade de Pernambuco, Recife, PE, Brasil \\ ${ }^{2}$ Departamento de Doenças Infecciosas e Parasitárias, \\ Faculdade de Medicina, Universidade de São Paulo, São Paulo, SP, Brasil \\ ${ }^{3}$ Departamento de Medicina Tropical, Centro de Ciências da Saúde, \\ Universidade Federal de Pernambuco, Recife, PE, Brasil
}

\author{
Correspondence \\ D.B. Miranda-Filho \\ Rua Cosme Bezerra, 85/107 \\ 50670-310 Recife, PE \\ Brasil \\ Fax: +55-81-3271-2880 \\ E-mail: demofilho@uol.com.br \\ Part of the data reported here were \\ presented at the XIII Brazilian \\ Meeting of Infectology, Goiânia, \\ GO, Brazil, August 31-September 3, \\ 2003. \\ Research supported by Fundação de \\ Amparo à Ciência e Tecnologia de \\ Pernambuco; Fundação de Saúde \\ Amaury de Medeiros/Secretaria de \\ Saúde do Estado de Pernambuco - \\ Projeto Nordeste; Centro Nacional \\ de Epidemiologia/Fundação Nacional \\ de Saúde/Ministério da Saúde do \\ Brasil.
}

Publication supported by FAPESP. $\ldots \ldots \ldots \ldots \ldots$

Received September 12, 2005

Accepted July 4, 2006

\begin{abstract}
The authors propose a clinical classification to monitor the evolution of tetanus patients, ranging from grade I to IV according to severity. It was applied on admission and repeated on alternate days up to the 10th day to patients aged $\geq 12$ years admitted to the State University Hospital, Recife, Brazil. Patients were also classified upon admission according to three prognostic indicators to determine if the proposed classification is in agreement with the traditionally used indicators. Upon admission, the distribution of the 64 patients among the different levels of the proposed classification was similar for the groups of better and worse prognosis according to the three indicators $(\mathrm{P}>0.05)$, most of the patients belonging to grades I and II of the proposed classification. In the later reclassifications, severe forms of tetanus (grades III and IV) were more frequent in the categories of worse prognosis and these differences were statistically significant. There was a reduction in the proportion of mild forms (grades I and II) of tetanus with time for the categories of worse prognostic indicators (chi-square for trend: $\mathrm{P}=0.00006,0.03$, and 0.00000 ) whereas no such trend was observed for the categories of better prognosis (grades I and II). This serially used classification reflected the prognosis of the traditional indicators and permitted the comparison of the dynamics of the disease in different groups. Thus, it becomes a useful tool for monitoring patients by determining clinical category changes with time, and for assessing responses to different therapeutic measures.
\end{abstract}

Key words

- Tetanus

- Progression

- Classification

- Clinical

\section{Introduction}

The concern with classifying tetanus patients is common among most investigators studying this subject. There are at least two practical reasons for this. First, the identification of groups of individuals with a potentially unfavorable prognosis by the observation of predictive factors, such as incubation period, period of onset, time between onset of symptoms and admission, and the type of wound through which the disease entered (1$10)$, is useful for the differentiated monitoring of groups at risk and for therapeutic planning (6). Secondly, it may be a tool for the determination of the clinical condition of tetanus patients at a specific time independently of what is suggested by prognostic indicators. These classifications are usually based on clinical manifestations $(2,11-18)$ 
and can be used to monitor the evolution of the disease after treatment has been initiated by comparing the classification established upon admission to reclassifications carried out throughout the hospital stay (16). However, there are as yet few studies that assess or validate and standardize a classification that encompasses the evolutional dynamics of tetanus.

The aim of the present study was to determine if a clinical classification proposed by the authors, applied upon admission to the hospital and repeated throughout the hospital stay, reflects what the traditional prognostic indicators suggest for tetanus.

\section{Material and Methods}

The study was conducted on tetanus patients admitted to the intensive care unit of the Oswaldo Cruz University Hospital, Recife, PE, Brazil. Patients aged 12 years or over and who had secondary sexual characteristics were included in the study. This investigation is part of a broader study, the objective of which is to evaluate the intrathecal route for the use of an anti-tetanus

Table 1. Prognostic indicators for tetanus applied on admission.

Prognostic classification of Armitage and Clifford (2)

Spasms at Time elapsed between the beginning admission of symptoms and admission

\begin{tabular}{lccc} 
& $0-36 \mathrm{~h}$ & $37-72 \mathrm{~h}$ & $>73 \mathrm{~h}$ \\
\hline Yes & $1 \mathrm{~A}$ & $2 \mathrm{~A}$ & $3 \mathrm{~A}$ \\
No & $2 \mathrm{~A}$ & $3 \mathrm{~A}$ & $3 \mathrm{~A}$ \\
\hline
\end{tabular}

Cut-off points of Miranda-Filho et al. (6)

Prognosis Incubation period Period of onset

\begin{tabular}{lll}
\hline Worse & $\leq 10$ days & $\leq 48 \mathrm{~h}$ \\
Better & $>10$ days & $>48 \mathrm{~h}$
\end{tabular}

Categories $1 \mathrm{~A}$ and $2 \mathrm{~A}$ of the Armitage and Clifford (2) classification indicate worse prognosis and category $3 \mathrm{~A}$ indicates good prognosis. immunoglobulin in the treatment of the disease. The group of patients who received conventional treatment (control group), plus 2 patients who were randomized to the intrathecal group but who received conventional treatment, due to technical problems, were selected for this study (19). The procedures followed were approved by the National Ethics Committee and were in accordance with the Helsinki Declaration. Written informed consent was obtained from the patients or persons responsible for them.

Upon admission, patients were classified according to the following prognostic indicators: incubation period, period of onset and the prognostic classification suggested by Armitage and Clifford (2) (Table 1). The selection of these indicators was based on the fact that they are well established in the tetanus literature. The cut-off point for incubation period and period of onset was based on a previous study in the same setting (6) and permitted the classification of the patients into two groups: worse (incubation period $\leq 10$ days, period of onset $\leq 48 \mathrm{~h}$ ) and better (incubation period $>10$ days, period of onset $>48 \mathrm{~h}$ ) prognosis. Categories $1 \mathrm{~A}$ and 2A of the Armitage and Clifford criteria (2) were combined to form the poor prognosis group and their category $3 \mathrm{~A}$ was equivalent to a good prognosis.

A second clinical classification proposed by the authors was applied upon admission to the hospital and repeated on alternate days to monitor the evolution of the disease until the 10th day of hospitalization. This classification considered the following clinical parameters: grade I, trismus + dysphagia + generalized rigidity (present in more than one segment - head, trunk, arms and legs - of the body), with no spasms; grade II, mild and occasional spasms (generally after a stimulus); grade III, severe and recurrent spasms, usually triggered by minor stimuli (light, sound, measurement of vital signs, light touch, opening the eyes) or imperceptible stimuli; grade IV, same features as 
grade III + syndrome of sympathetic nervous system hyperactivity.

The difference between grades II and III was based on the frequency and intensity of the spasms and on how easily which spasms could be evoked or triggered. Grade II patients include those with at least two or three characteristics of minor severity in the description of spasms (mild, occasional, triggered); grade III patients are those with at least two severe characteristics (intense, frequent, spontaneous spasms). Grade IV differed from grade III by the presence of hyperactivity of the sympathetic nervous system in the former group. To characterize the presence of hyperactivity of the sympathetic nervous system, the most frequent signs and symptoms were grouped into two categories: major signs (oscillation of arterial pressure, arrhythmia and oscillation of heart rate), and lesser signs (generalized sweating, paralytic ileum and other signs not described on the form, but that could be attributed to the condition). The presence of at least two major signs, or one major sign and two minor signs, was taken to indicate sympathetic nervous system hyperactivity.

The clinical classification was repeated on alternate days to monitor the evolution of the disease throughout the 10th day of hospitalization. Patients were observed with regard to whether there were changes from one severity category to another, characterizing improvement, deterioration or stabilization. For the analysis of this variable, the patients who died within the first 10 days of hospitalization were considered to belong to the deterioration category (unfavorable evolution), along with those having changed from one clinical stage to another of greater severity. Patients who exhibited improvement, that is, changing from one clinical form to another of lesser severity between the 2nd and 5th day of hospital stay, were grouped into the "progressive improvement" category. Those who were stable in relation to tetanus and only improved after the 6th day were grouped into the third category (stabilization).

The results are summarized, with a description of the clinical classification of patients on the 2nd, 6th, and 10th days of hospitalization.

\section{Data collection}

A standardized individual form was employed to collect information on the progression and outcome of each case, as well as outpatient re-evaluation. In addition, weekly meetings were held for discussion and confirmation of these criteria.

Different approaches were utilized in order to minimize observation bias: a) the use of the above-mentioned standardized form; b) rotation among the doctors involved in the clinical classification of patients (carried out every other day after admission throughout the 10th day); c) the clinical classification was recorded on an additional individual form which contained no information on the patient's treatment or on previous classifications performed by other doctors; d) periodic meetings with the team were held to discuss doubts.

\section{Data processing and analysis}

Double entries of the data were made, using the Epi-Info 6.0 software for data analysis. The frequency of each outcome in the groups of worse and better prognosis, for each classic prognostic indicator utilized, was compared by the chi-square test. The chi-square test for linear trend of proportions was used for ordered categories.

\section{Results}

A total of 64 patients were studied. The clinical classification of all patients at admission and at days 2,6 , and $10\left(\mathrm{D}_{2}, \mathrm{D}_{6}\right.$, and $\mathrm{D}_{10}$, respectively) is shown in Table 2. Figures 1,2 , and 3 show the clinical evolution 
Table 2. Sequential clinical classification of tetanus patients and their evolution from admission $\left(D_{0}\right)$ to the 10th day $\left(D_{10}\right)$ of hospitalization.

\begin{tabular}{|c|c|c|c|c|c|c|c|c|c|}
\hline \multirow[t]{2}{*}{ Patient No. } & \multirow[t]{2}{*}{ Age } & \multirow[t]{2}{*}{ Sex } & \multirow[t]{2}{*}{$\begin{array}{l}\text { Incubation } \\
\text { period (days) }\end{array}$} & \multirow[t]{2}{*}{$\begin{array}{l}\text { Period of } \\
\text { onset }(\mathrm{h})\end{array}$} & \multirow[t]{2}{*}{$\begin{array}{l}\text { Armitage and } \\
\text { Clifford criteria }\end{array}$} & \multicolumn{4}{|c|}{$\begin{array}{c}\text { Grade } \\
\text { (clinical classification) }\end{array}$} \\
\hline & & & & & & $\mathrm{D}_{0}$ & $\mathrm{D}_{2}$ & $\mathrm{D}_{6}$ & $\mathrm{D}_{10}$ \\
\hline 1 & 23 & $M$ & $\leq 10$ & $\leq 48$ & $3 A$ & III & III & III & III \\
\hline 2 & 22 & $M$ & $\lg n$ & $>48$ & $3 \mathrm{~A}$ & 1 & 1 & II & 1 \\
\hline 3 & 58 & $M$ & $>10$ & $>48$ & $3 \mathrm{~A}$ & II & III & III & II \\
\hline 4 & 67 & $M$ & $>10$ & $>48$ & $2 A$ & 1 & 1 & II & I \\
\hline 5 & 65 & $\mathrm{~F}$ & $\lg n$ & $\leq 48$ & $3 A$ & III & III & II & ॥ \\
\hline 6 & 49 & $M$ & $\leq 10$ & $\lg n$ & $3 A$ & ॥ & 1 & I & II \\
\hline 7 & 61 & $\mathrm{~F}$ & $\leq 10$ & $\leq 48$ & $1 \mathrm{~A}$ & 1 & III & III & * \\
\hline 8 & 19 & $M$ & $\leq 10$ & $\leq 48$ & $1 \mathrm{~A}$ & I & IV & IV & IV \\
\hline 9 & 18 & $M$ & $\leq 10$ & $\leq 48$ & $1 \mathrm{~A}$ & ॥ & III & IV & IV \\
\hline 10 & 82 & $M$ & $>10$ & $>48$ & $3 A$ & III & 1 & 1 & PD \\
\hline 11 & 42 & $M$ & $\lg n$ & $\leq 48$ & $2 \mathrm{~A}$ & ॥ & IV & III & III \\
\hline 12 & 62 & $\mathrm{~F}$ & $\operatorname{lgn}$ & $>48$ & $2 \mathrm{~A}$ & II & III & IV & III \\
\hline 13 & 36 & $M$ & $\leq 10$ & $\leq 48$ & $1 \mathrm{~A}$ & II & III & IV & III \\
\hline 14 & 83 & $\mathrm{~F}$ & $\lg n$ & $>48$ & $3 A$ & III & I & NE & 1 \\
\hline 15 & 80 & $M$ & $\leq 10$ & $\lg n$ & $1 \mathrm{~A}$ & 1 & * & * & * \\
\hline 16 & 67 & $\mathrm{~F}$ & $>10$ & $>48$ & $3 A$ & II & 1 & I & PD \\
\hline 17 & 25 & $M$ & $\leq 10$ & $\lg n$ & $3 A$ & I & $\mathrm{NE}$ & PD & PD \\
\hline 18 & 23 & $M$ & $\leq 10$ & $\operatorname{lgn}$ & $2 A$ & 1 & 1 & PD & PD \\
\hline 19 & 29 & $M$ & $\leq 10$ & $\leq 48$ & $2 \mathrm{~A}$ & III & IV & III & III \\
\hline 20 & 28 & $M$ & $\leq 10$ & $>48$ & $3 A$ & III & III & III & $\mathrm{NE}$ \\
\hline 21 & 44 & $M$ & $\leq 10$ & $\leq 48$ & $2 \mathrm{~A}$ & II & III & III & III \\
\hline 22 & 37 & $M$ & $\lg n$ & $\leq 48$ & $3 \mathrm{~A}$ & III & III & IV & III \\
\hline 23 & 66 & $M$ & $\leq 10$ & $>48$ & $2 \mathrm{~A}$ & II & IV & III & IV \\
\hline 24 & 22 & $M$ & $>10$ & $\lg n$ & $2 \mathrm{~A}$ & 1 & 1 & PD & PD \\
\hline 25 & 64 & $\mathrm{~F}$ & $\leq 10$ & $>48$ & $2 \mathrm{~A}$ & 1 & II & * & * \\
\hline 26 & 19 & $M$ & $\leq 10$ & $\lg n$ & $3 A$ & 1 & 1 & 1 & PD \\
\hline 27 & 54 & $M$ & $>10$ & $\leq 48$ & $3 A$ & II & II & III & II \\
\hline 28 & 48 & $M$ & $\leq 10$ & $>48$ & $3 A$ & 1 & II & 1 & II \\
\hline 29 & 65 & $M$ & $>10$ & $>48$ & $3 A$ & 1 & I & II & II \\
\hline 30 & 73 & $M$ & $\leq 10$ & $\leq 48$ & $2 A$ & II & * & * & * \\
\hline 31 & 84 & $M$ & $>10$ & $>48$ & $3 A$ & I & NE & $\mathrm{NE}$ & * \\
\hline 32 & 20 & $\mathrm{~F}$ & $\leq 10$ & $\leq 48$ & $3 \mathrm{~A}$ & III & NE & IV & III \\
\hline 33 & 35 & $M$ & $>10$ & $>48$ & $3 A$ & 1 & II & 1 & PD \\
\hline 34 & 66 & $\mathrm{~F}$ & $>10$ & $\leq 48$ & $2 \mathrm{~A}$ & III & III & III & III \\
\hline 35 & 31 & $M$ & $\leq 10$ & $\leq 48$ & $2 \mathrm{~A}$ & III & III & IV & III \\
\hline 36 & 61 & $M$ & $\leq 10$ & $\leq 48$ & $1 \mathrm{~A}$ & 1 & II & IV & III \\
\hline 37 & 79 & $M$ & $\leq 10$ & $>48$ & $3 A$ & 1 & 1 & III & III \\
\hline 38 & 51 & $M$ & $>10$ & $>48$ & $3 \mathrm{~A}$ & II & II & II & 1 \\
\hline 39 & 46 & $M$ & $\leq 10$ & $\leq 48$ & $1 \mathrm{~A}$ & II & II & II & II \\
\hline 40 & 25 & $M$ & $>10$ & $\leq 48$ & $2 \mathrm{~A}$ & III & III & II & II \\
\hline 41 & 33 & $M$ & $\lg n$ & $\leq 48$ & $3 \mathrm{~A}$ & III & IV & III & III \\
\hline 42 & 65 & $M$ & $\leq 10$ & $\leq 48$ & $2 \mathrm{~A}$ & III & III & III & III \\
\hline 43 & 25 & $M$ & $>10$ & $\leq 48$ & $2 A$ & II & IV & IV & IV \\
\hline 44 & 39 & $M$ & $\leq 10$ & $>48$ & $2 \mathrm{~A}$ & 1 & II & III & $\mathrm{NE}$ \\
\hline 45 & 35 & $M$ & $\leq 10$ & $\leq 48$ & $2 \mathrm{~A}$ & 1 & IV & IV & III \\
\hline 46 & 43 & $M$ & $\leq 10$ & $\lg n$ & $3 A$ & 1 & 1 & 1 & I \\
\hline 47 & 14 & $\mathrm{~F}$ & $\leq 10$ & $\leq 48$ & $2 A$ & II & IV & IV & IV \\
\hline 48 & 57 & $M$ & $\leq 10$ & $\leq 48$ & $2 \mathrm{~A}$ & II & III & $\mathrm{NE}$ & III \\
\hline 49 & 32 & $M$ & $>10$ & $\lg n$ & $2 \mathrm{~A}$ & 1 & 1 & I & PD \\
\hline
\end{tabular}


Table 2 continued.

\begin{tabular}{|c|c|c|c|c|c|c|c|c|c|}
\hline \multirow[t]{2}{*}{ Patient No. } & \multirow[t]{2}{*}{ Age } & \multirow[t]{2}{*}{ Sex } & \multirow[t]{2}{*}{$\begin{array}{l}\text { Incubation } \\
\text { period (days) }\end{array}$} & \multirow[t]{2}{*}{$\begin{array}{l}\text { Period of } \\
\text { onset }(h)\end{array}$} & \multirow[t]{2}{*}{$\begin{array}{l}\text { Armitage and } \\
\text { Clifford criteria }\end{array}$} & \multicolumn{4}{|c|}{$\begin{array}{c}\text { Grade } \\
\text { (clinical classification) }\end{array}$} \\
\hline & & & & & & $\mathrm{D}_{0}$ & $\mathrm{D}_{2}$ & $\mathrm{D}_{6}$ & $D_{10}$ \\
\hline 50 & 64 & M & $>10$ & $\operatorname{lgn}$ & $3 A$ & 1 & 1 & 1 & PD \\
\hline 51 & 32 & $M$ & $\lg n$ & $>48$ & $3 A$ & I & III & IV & IV \\
\hline 52 & 48 & $M$ & $>10$ & $\leq 48$ & $3 A$ & II & II & II & NE \\
\hline 53 & 22 & M & $>10$ & $>48$ & $3 A$ & 1 & II & II & NE \\
\hline 54 & 22 & M & $>10$ & $>48$ & $3 A$ & II & NE & 1 & PD \\
\hline 55 & 59 & $M$ & $>10$ & $>48$ & $3 A$ & I & II & II & II \\
\hline 56 & 39 & M & $\leq 10$ & $>48$ & $2 A$ & II & II & III & II \\
\hline 57 & 27 & $M$ & $\leq 10$ & $>48$ & $3 A$ & III & III & III & III \\
\hline 58 & 20 & $F$ & $>10$ & $>48$ & $3 A$ & II & II & 1 & 1 \\
\hline 59 & 30 & $M$ & $>10$ & $\leq 48$ & $2 A$ & I & 1 & NE & I \\
\hline 60 & 49 & M & $>10$ & $\leq 48$ & $3 A$ & II & 1 & 1 & 1 \\
\hline 61 & 25 & $M$ & $\leq 10$ & $\leq 48$ & $3 A$ & II & 1 & I & PD \\
\hline 62 & 29 & M & $\leq 10$ & $>48$ & $2 \mathrm{~A}$ & II & III & III & NE \\
\hline 63 & 57 & $M$ & $\operatorname{lgn}$ & $\leq 48$ & $3 A$ & III & III & II & II \\
\hline 64 & 20 & $M$ & $\leq 10$ & $\leq 48$ & $3 \mathrm{~A}$ & III & NE & II & 1 \\
\hline
\end{tabular}

See Material and Methods for explanation of clinical classification (grades I-IV). Categories $1 \mathrm{~A}$ and $2 \mathrm{~A}$ of the Armitage and Clifford (2) classification indicate worse prognosis and category $3 \mathrm{~A}$ indicates good prognosis. Based on Miranda-Filho et al. (6), incubation period $\leq 10$ days and period of onset $\leq 48 \mathrm{~h}$ indicate worse prognosis and incubation period $>10$ days and period of onset $>48 \mathrm{~h}$ indicate better prognosis.

Ign = ignored; *death; PD = patient discharged; NE = not evaluated.
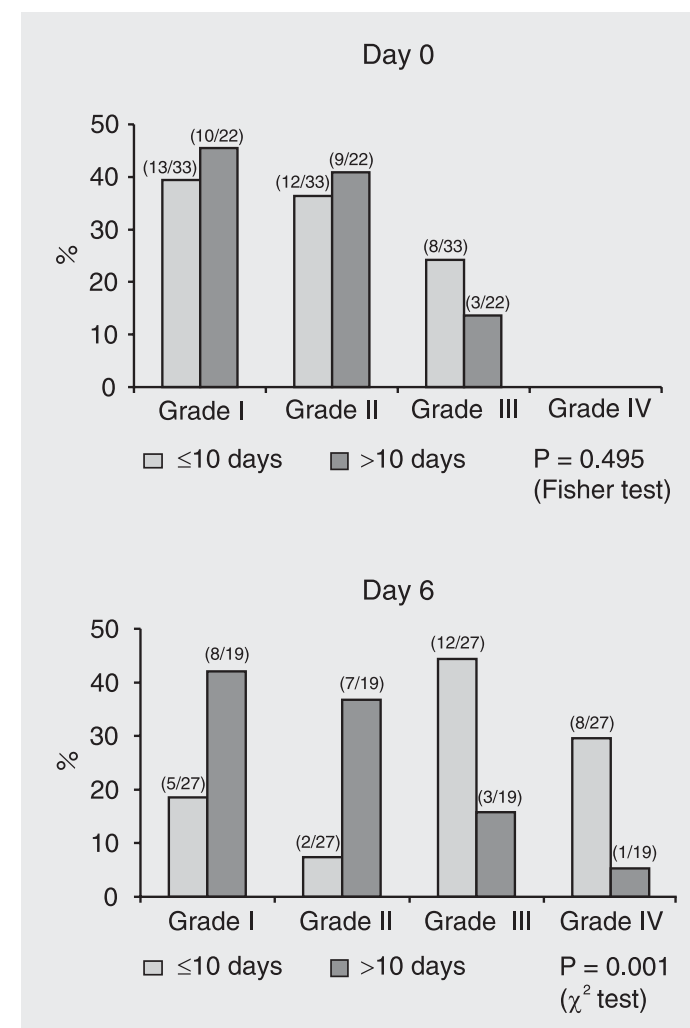
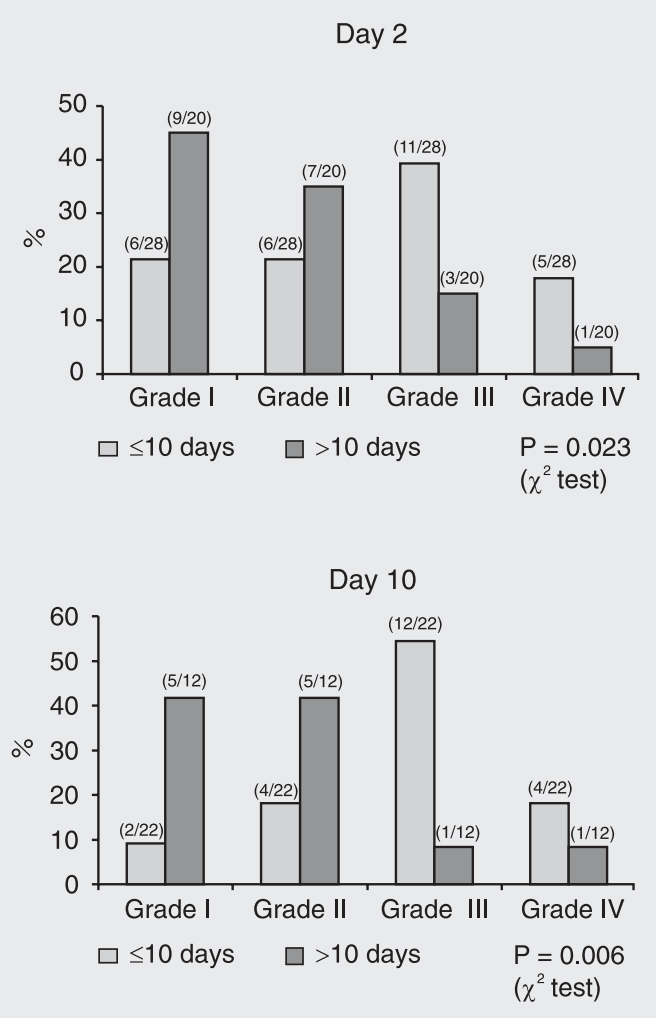

Figure 1. Clinical classification of tetanus patients upon admission (day 0 ) and on the 2nd, 6th, and 10 th days of hospital stay, according to the incubation period. The numbers included in day 0 vary because of variable numbers with missing information for each variable. For statistical analysis, patients were regrouped into two categories: one with those classified as grades I and II, and the other with patients classified as grades III and IV. Cut-off point for the incubation period was based on Miranda-Filho et al. (6); $\leq 10$ days indicating worse prognosis and $>10$ days indicating better prognosis. 
Figure 2. Clinical classification of tetanus patients upon admission (day 0) and on the 2nd, 6th, and 10th days of hospital stay, according to the period of onset. The numbers included in day 0 vary because of variable numbers with missing information for each variable. For statistical analysis, patients were regrouped into two categories: one with those classified as grades I and II, and the other with patients classified as grades III and IV. Cut-off point for the period of onset was based on MirandaFilho et al. (6); $\leq 48 \mathrm{~h}$ indicating worse prognosis and $>48 \mathrm{~h}$ indicating better prognosis.
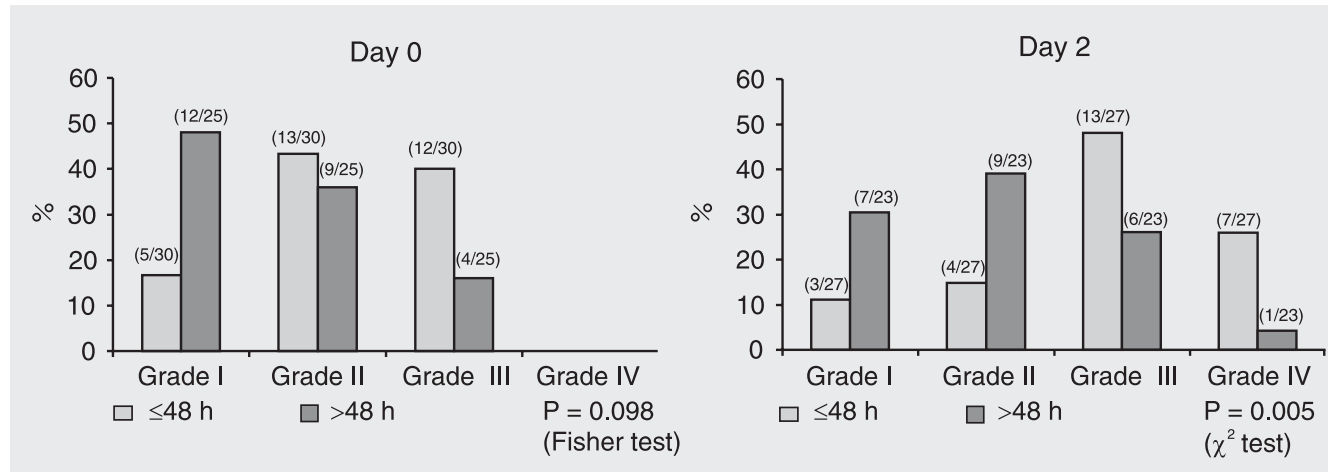

Day 6

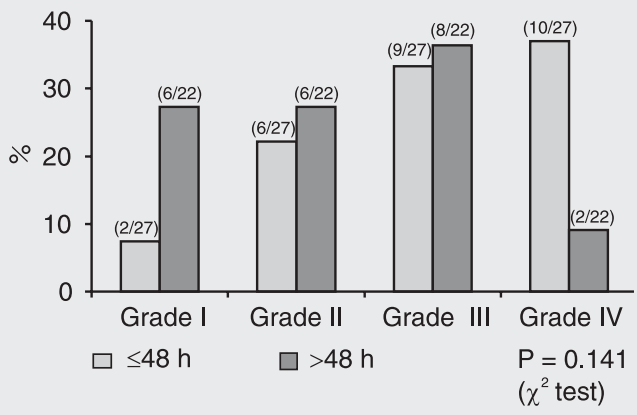

Day 10

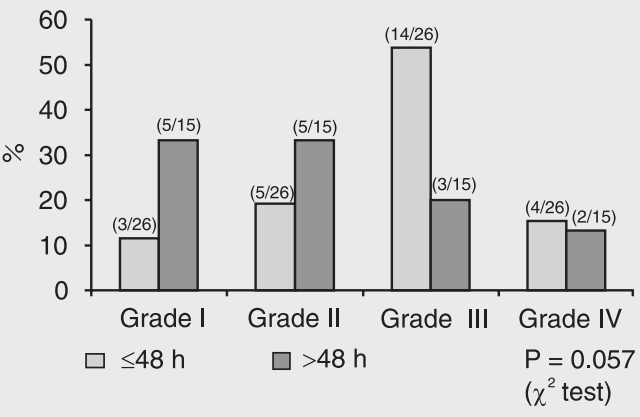

Figure 3. Clinical classification of tetanus patients upon admission (day 0) and on the 2nd, 6th, and 10th days of hospital stay, according to the prognostic classification by Armitage and Clifford (2). For statistical analysis, patients were regrouped into two categories: one with those classified as grades I and II, and the other with patients classified as grades III and IV.
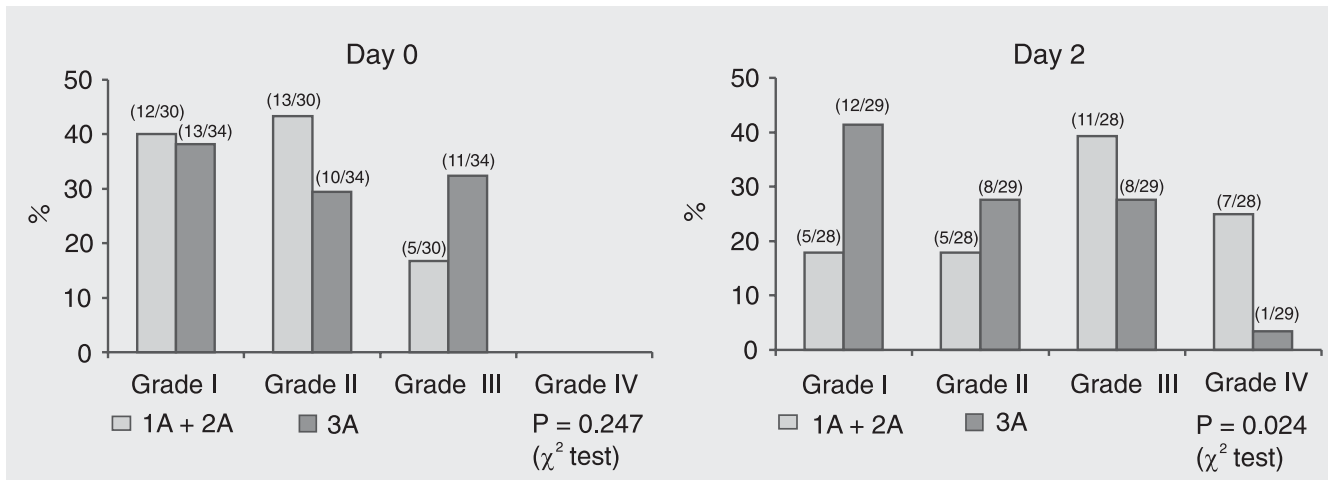

Day 6

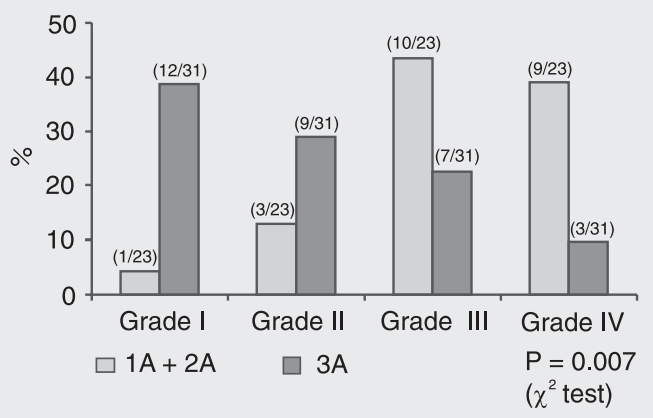

Day 10

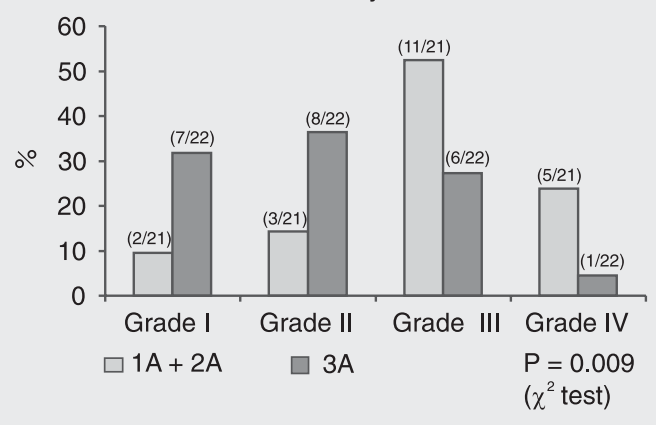


of patients in each group of the classic prognostic indicators. In general, in the successive evaluations, a higher frequency of patients with a more severe form of tetanus was observed in the groups of worse prognosis for each indicator.

Upon admission $\left(\mathrm{D}_{0}\right)$, distribution of patients among the different levels of the authors' clinical classification was similar for the groups of better and worse prognosis according to the three classic prognostic indicators utilized $(\mathrm{P}>0.05)$. The data regarding the evaluation of patients on successive days (Figures 1, 2, and 3) show the changes in the percent distribution of individuals among the four grades of the clinical classification, indicating a relative increase of more severe forms (grades III and IV) along time in the groups of worse prognosis, which was not observed in the groups of better prognosis.

Results related to each classic prognostic indicator are presented below: 1) Incubation period: on days 2,6 , and 10 there was a greater proportion of patients with severe forms of the disease (grades III and IV) in the category of worse prognosis (incubation period $\leq 10$ days). This difference was statistically significant, with $\mathrm{P}=0.023, \mathrm{P}=0.006$ and 0.001, respectively (Figure 1).2) Period of onset: on days 2,6 , and 10 there was a predominance of the more severe clinical forms of tetanus (grades III and IV) in the category of worse prognosis (period of onset $\leq 48$ h), with $\mathrm{P}=0.005,0.14$, and 0.057 , respectively. The difference was statistically significant on $\mathrm{D}_{2}$ and the level of significance was borderline on $\mathrm{D}_{10}$ (Figure 2). 3) Armitage and Clifford criteria: severe forms of tetanus (grades III and IV) were found more frequently in the category of worse prognosis (1A and 2A). This difference was statistically significant at all clinical reclassification times $\left(\mathrm{D}_{2}, \mathrm{D}_{6}\right.$ and $\left.\mathrm{D}_{10}\right)$, with values of $\mathrm{P}=0.02,0.0007$, and 0.009 , respectively (Figure 3).

Analysis of linear trend of proportions showed that the reduction in the proportion of mild forms (grades I and II) of tetanus over time (from $D_{0}$ to $D_{10}$ ) was statistically significant for the three categories of worse prognosis (chi-square for trend: incubation period $\leq 10$ days $(\mathrm{P}=0.00006)$, period of onset $\leq 48 \mathrm{~h}(\mathrm{P}=0.03)$ and $1 \mathrm{~A}+2 \mathrm{~A}(\mathrm{P}=$ $0.00000)$, whereas the results suggested no such trend for the categories of better prognosis.

The proposed classification criteria were also used to summarize the clinical progression of tetanus, grouping patients into different evolution categories. They demonstrated stabilization followed by improvement in 12 (18.8\%), progressive improvement in 13 (20.3\%) and deterioration in 37 (57.8\%) of the 64 patients analyzed, excluding two for which no information was obtained.

\section{Discussion}

It is known that interventions currently adopted in the treatment of severe diseases such as tetanus tend to be more effective due to technological advances and improved care in intensive care units, resulting in an important reduction of lethality in places where such resources are available $(11,14,20-22)$. Death from tetanus can vary from 6 to $60 \%$ depending on the places where treatment is provided, the severity of the disease and the therapeutic measures adopted $(8,10,20,22$, 23). In hospitals where death from tetanus is low due to the reasons cited above, comparing this indicator (death from tetanus) alone among the groups of patients subjected to different treatments requires very large samples. In such places, the incidence of the disease is generally lower than in poorer regions where medical assistance is also precarious. For this reason, it may be difficult, or even impracticable, to conduct clinical trials in which death from tetanus is the main outcome in some places.

Different investigators have used a number of indicators of morbidity and on the 
evolution of the disease. The most frequently cited are duration of hospitalization, length of stay in the intensive care unit, the need for and the duration of respiratory assistance, frequency of complications, the clinical progression of the disease, the need for tracheotomy, and the sedative dose needed to control spasms, as well as lethality $(1,8,16,19,24-$ 30).

Assessing the clinical evolution of tetanus patients involves difficulties due to the subjectivity of the criteria present in most of the systems of classifications used, as well as clinical fluctuations that an individual might exhibit in a single day stemming from the need to handle the patient, the dosage and intervals of sedatives being employed, the presence of spasm-triggering factors, and the occurrence of complications or associated diseases. In assessing a therapeutic measure regarding diseases with a high potential for morbimortality such as tetanus, one should take into account the potential benefits that treatment can bring regarding the reduction of patient suffering, treatment costs, the duration of symptoms, duration of hospitalization, and the risk of complications or sequelae, as well as the possibility of reducing lethality.

In the present study, most of the patients (75\% of the cases), upon admission to the hospital, were classified as having grade $\mathrm{I}(\mathrm{N}$ $=25)$ or II $(\mathrm{N}=23)$ tetanus according to the proposed clinical classification.

The distribution of the patients among the four categories of the proposed clinical classification in the group of worse and better prognosis was similar upon admission $\left(D_{0}\right)$. In all of the reclassifications $\left(D_{2}, D_{6}\right.$, and $\mathrm{D}_{10}$ ), the severe forms of tetanus (grades III and IV) were present more frequently in the category of worse prognosis using the Armitage and Clifford criteria (1A and 2A). A larger proportion of the more severe clinical forms of tetanus (grades III and IV) was also detected in the category of worse prognosis with regard to the progression period (period of onset $\leq 48 \mathrm{~h}$ ) on $\mathrm{D}_{2}, \mathrm{D}_{6}$ and $\mathrm{D}_{10}$, being statistically significant on $\mathrm{D}_{2}$ and having a $\mathrm{P}$ value of borderline significance on $\mathrm{D}_{10}$. Concerning the incubation period, a difference was observed on $\mathrm{D}_{2}, \mathrm{D}_{6}$ and $\mathrm{D}_{10}$, with a greater proportion of patients with severe forms of tetanus (grades III and IV) in the category of worse prognosis (incubation period $\leq 10$ days).

The analysis of linear trend for proportion suggested that there was a constant reduction in the proportion of mild forms (grades I and II) of tetanus with time, for the three categories of worse prognostic indicators (incubation period $\leq 10$ days, period of onset $\leq 48 \mathrm{~h}$ and $1 \mathrm{~A}+2 \mathrm{~A}$ ).

The clinical evolution of tetanus patients evaluated with objective criteria, such as those proposed in the present paper, allows comparisons between patients or groups. The classification system proposed here can be used to characterize cases of favorable evolution (progressive improvement or stabilization followed by improvement) as well as unfavorable evolution through the determination of clinical category changes at predetermined times. The sequential use of the proposed classification allows follow-up of the evolutional dynamics of tetanus and reflects the prognosis of indicators used upon admission to the hospital, providing a useful tool for monitoring patients and for assessing responses to different therapeutic measures. The proposed classification was used in a clinical trial of tetanus treatment with antitetanus immunoglobulin by the intrathecal or intramuscular route to evaluate the effect of such therapy on clinical progression of and mortality from tetanus (19).

The objective of the clinical classification proposed by the authors is to monitor tetanus progression. Different from prognostic indicators, which aim at the early identification of higher risk subgroups, this classification provides a rapid snapshot at different moments in time and, thus, to map the evolution of tetanus patients. 
The use of the sequential clinical classification permitted the determination and comparison of the dynamics of the disease in different groups. Thus, this classification is a useful tool for monitoring patients by the determination of changes in clinical category at predetermined times, and for assessing responses to different therapeutic measures. Although simple, the analysis meets its proposed objective.

\section{References}

1. Agarwal M, Thomas K, Peter JV, Jeyaseelan L, Cherian AM. A randomized double-blind sham-controlled study of intrathecal human anti-tetanus immunoglobulin in the management of tetanus. Natl Med J India 1998; 11: 209-212.

2. Armitage $P$, Clifford R. Prognosis in tetanus: Use of data from therapeutic trials. J Infect Dis 1978; 138: 1-8.

3. Bleck TP. Tetanus: pathophysiology, management, and prophylaxis. Dis Mon 1991; 37: 545-603.

4. Cole L, Youngman H. Treatment of tetanus. Lancet 1969; 1: 10171019.

5. Debord T, Lapeyre E. Tétanos. In: Anonymous (Editors), Encyclopédie médico-chirurgicale. Maladies infectieuses. Paris: Elsevier; 1995. p 1-6.

6. Miranda-Filho DB, Ximenes RA, Bernardino SN, Escariao AG. Identification of risk factors for death from tetanus in Pernambuco, Brazil: a case-control study. Rev Inst Med Trop Sao Paulo 2000; 42: 333339.

7. Patel JC, Mehta BC. Tetanus: study of 8.697 cases. Proceedings of the International Conference on Tetanus, Lyon. Lyon: Fondation Mérieux; 1975. p 233-241.

8. Sanders RK, Martyn B, Joseph R, Peacock ML. Intrathecal antitetanus serum (horse) in the treatment of tetanus. Lancet 1977; 1: 974977.

9. Sanders RK. The management of tetanus 1996. Trop Doct 1996; 26 : 107-115.

10. Veronesi R, Focaccia R, Tavares W, Mazza CC. Tétano. In: Veronesi R, Focaccia R (Editors), Tratado de infectologia. São Paulo: Atheneu; 1996. p 887-913.

11. Attygalle D, Karalliedde L. Unforgettable tetanus. Eur J Anaesthesiol 1997; 14: 122-133.

12. Barone AA, Raineri HC, Ferreira JM. Tetanus: its epidemiological, clinical and therapeutic aspects. Analysis of 461 cases. Rev Hosp Clin Fac Med Sao Paulo 1976; 31: 215-225.

13. Betancur J, Gomez L, Castellanos R. Tétanos. Acta Med Colomb 1987; 12: 289-293.

14. Edmondson RS, Flowers MW. Intensive care in tetanus: management, complications, and mortality in 100 cases. Br Med J 1979; 1 : 1401-1404.

15. Focaccia R. Tétano. In: Farhat CK, Carvalho ES, Carvalho KLHFR, Succi RCM (Editors), Infectologia pediátrica. São Paulo: Atheneu; 1993. p 207-213.

16. Gupta PS, Kapoor R, Goyal S, Batra VK, Jain BK. Intrathecal human tetanus immunoglobulin in early tetanus. Lancet 1980; 2: 439-440.
17. Khajehdehi $P$, Rezaian GR. Tetanus in the elderly: is it different from that in younger age groups? Gerontology 1998; 44: 172-175.

18. Tavares W. Infecções e trauma - I. Tétano. In: Shechter M, Marangoni DV (Editors), Doenças infecciosas - condutas diagnóstica e terapêutica. Rio de Janeiro: Guanabara Koogan; 1994. p 387-392.

19. Miranda Filho DB, Ximenes RAA, Barone AA, Vaz VL, Vieira AG, Albuquerque VMG. Randomised controlled trial of tetanus treatment with antitetanus immunoglobulin by the intrathecal or intramuscular route. Br Med J 2004; 328: 615-617.

20. Bleck TP. Clostridium tetani (Tetanus). In: Mandell GL, Bennett JE, Dolin R (Editors), Mandell, Douglas and Bennett's principles and practice of infectious diseases. 6th edn. Philadelphia: Churchill Livingstone; 2004. p 2817-2822.

21. Jolliet P, Magnenat JL, Kobel T, Chevrolet JC. Aggressive intensive care treatment of very elderly patients with tetanus is justified. Chest 1990; 97: 702-705.

22. Nolla-Salas M, Garces-Bruses J. Severity of tetanus in patients older than 80 years: comparative study with younger patients. Clin Infect Dis 1993; 16: 591-592.

23. Faust RA, Vickers OR, Cohn I Jr. Tetanus: 2,449 cases in 68 years at Charity Hospital. J Trauma 1976; 16: 704-712.

24. Ahmadsyah I, Salim A. Treatment of tetanus: an open study to compare the efficacy of procaine penicillin and metronidazole. $\mathrm{Br}$ Med J 1985; 291: 648-650.

25. Keswani NK, Singh AK, Upadhyaya KD. Intrathecal tetanus antitoxin in moderate and severe tetanus. J Indian Med Assoc 1980; 75: 67-69.

26. List WF. The immediate treatment of tetanus with high doses of human tetanus antitoxin. Notfallmedizin 1981; 7: 731-733.

27. Sun KO, Chan YW, Cheung RT, So PC, Yu YL, Li PC. Management of tetanus: a review of 18 cases. J R Soc Med 1994; 87: 135-137.

28. Vakil BJ, Armitage P, Clifford RE, Laurence DR. Therapeutic trial of intracisternal human tetanus immunoglobulin in clinical tetanus. Trans R Soc Trop Med Hyg 1979; 73: 579-583.

29. Veronesi R, Bizzini B, Hutzler RU, Focaccia R, Mazza CC, Feldman C, et al. Eficácia do tratamento do tétano com antitoxina tetânica por via raquideana e/ou venosa. Estudo de 101 casos, com pesquisa sobre a permanência da gamaglobulina humana - $F(a b)^{2}$ - no líqüor e no sangue. Rev Bras Clin Terap 1980; 9: 301-319.

30. Veronesi R, Bizzini B, Mazza CC, Focaccia R, Feldman C, Coscina $A L$, et al. Specific treatment of tetanus with a fraction - $F(a b$ ') - of the antitetanus immunoglobulin injected intrathecally. Rev Hosp Clin Fac Med São Paulo 1983; 38: 147-149. 